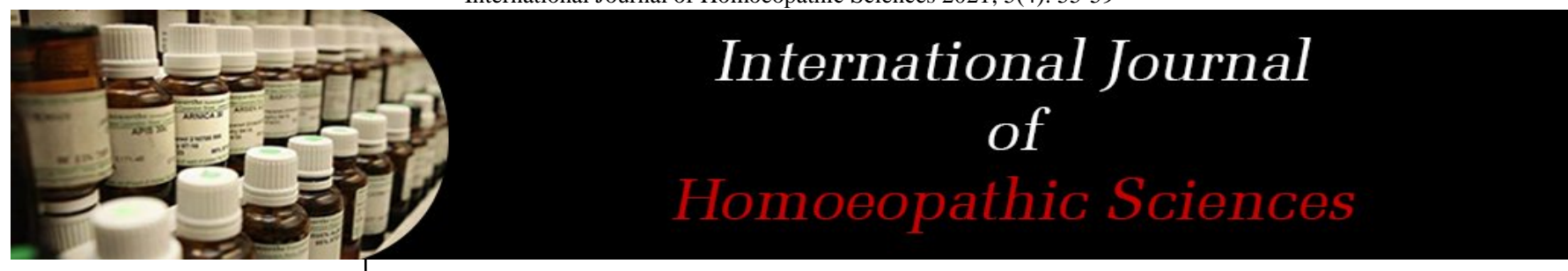

E-ISSN: 2616-4493 P-ISSN: 2616-4485 www.homoeopathicjournal.com IJHS 2021; 5(4): 55-59

Received: 28-08-2021

Accepted: 30-09-2021

Dr. Susy Charles M.D. Hom. PhD, Associate professor, Department of Materia Medica, Dr. Padiar Memorial Homoeopathic Medical College Chottanikkara, Ernakulam, Kerala, India

\section{Adenomyomatosis of Gallbladder: A case study with Constitutional Homoeopathic treatment}

\author{
Dr. Susy Charles
}

DOI: https://doi.org/10.33545/26164485.2021.v5.i4a.454

\begin{abstract}
Adenomyomatosis of Gallbladder is characterised by hypertrophy of the mucosal epithelium and the invagination of this epithelium into the interstices of a thickened muscularis, forming sinuses called Rokitansky-Aschoff sinuses. Three forms of adenomyomatosis are found in gallbladder; segmental, fundal and diffuse. Symptomatic adenomyomatosis is an indication for surgical intervention ${ }^{[1]}$. A seventeen year old girl presented with a history of recurrent attack of upper abdominal pain of two years duration.Ultrasonography of abdomen shows the features of adenomyomatosis of gallbladder. After detailed case taking, analysis, evaluation and repertorisation Pulsatilla was given. Abdominal pain was completely relieved and ultrasound scan report of abdomen after homoeopathic treatment shows normal gallbladder contour. This result shows that constitutional homoeopathic treatment is effective in the management of Adenomyomatosis of gallbladder.
\end{abstract}

Keywords: Gallbladder Adenomyomatosis, Rokitansky- Aschoff sinuses, Cholecystectomy, Pulsatilla, Constitutional homoeopathic remedy

\section{Introduction}

Adenomyomatosis of gallbladder is a benign condition and it is characterised by hyperplasic changes involving the gallbladder wall and overgrowth of the mucosa, thickening of the muscular wall, and formation of sinus tracts termed Rokitansky-Aschoff sinus ${ }^{[2,3]}$.

\subsection{Epidemiology}

Incidence of Gallbladder adenomyomatosis (GA) increases with age and usually a female predilection $(\mathrm{M}: \mathrm{F}=1: 3)^{[15]}$. Females are more predisposed to gallbladder diseases, including gallstones, adenomyomatosis and gallbladder carcinoma ${ }^{[4]}$. Aetiology of adenomyomatosis is unknown ${ }^{[5]}$.

\subsection{Pathology}

Adenomyomatosis lesion of the Gallbladder is with no malignant potential and may involve the Gallbladder in three forms called focal (fundal), segmental and more rarely diffuse. Most common type is focal and usually involves the gallbladder fundus ${ }^{[2]}$. In fundal GA, focal thickening involving gallbladder fundus and in segmental GA, there is circumferential over growth of the gallbladder wall that leads to formation of compartments. In diffuse GA, disseminated thickening and irregularity of the mucosa and muscularis are found [6]. Rokitasky-Aschoff sinuses (RAS) are characterised by microscopic or macroscopic pseudo diverticular or pockets in the gallbladder. Histologically, they are present as out pouching of gallbladder mucosa into the gallbladder muscle layer and subserosal tissue and these results in hyperplasia and herniation of epithelial cells into the fibromuscular layer of the gall bladder wall ${ }^{[7]}$.

\subsection{Clinical features}

The duration of symptoms of GA may vary from a few days to months. Most common presentation of GA is abdominal pain with intermittent bouts of right upper quadrant pain similar to symptomatic gallbladder disease. Nausea and vomiting, intolerance to fatty food are also reported symptoms ${ }^{[8]}$. The clinical presentation mimics, the more common conditions such as cholecystitis, choledocholelithiasis, cancer of the bile duct and pancreatic cancer.
Dr. Susy Charles

M.D. Hom. PhD, Associate professor, Department of Materia Medica, Dr. Padiar Memorial Homoeopathic Medical College Chottanikkara, Ernakulam, Kerala, India 


\subsection{Diagnosis}

A correct diagnosis of GA is neccessary in order to avoid unnecessary cholecystectomies. Ultrasound scan is the imaging modality of choice for diagnosing adenomyomatosis ${ }^{[9]}$. Usually sonographically evident gallbladder wall thickening has its aetiology in inflammatory or neoplastic conditions, with cholecystitis. The diffuse wall thickening with intramural diverticula forming the "Rosary sign" and "Pearl necklace sign" and hourglass configuration of the gallbladder usually confirm the radiological diagnosis ${ }^{[10]}$. GA is uncommon and need to be distinguished from other similar conditions such as choledocholelithiasis, sclerosing cholangitis, carolis disease and choledochal cyst.

\subsection{Treatment}

Symptomatic adenomyomatosis especially abdominal pain or hepatic colic even in the absence of gallstones is an indication for cholecystectomy. Surgical intervention is the first choice of treatment for patients with $\mathrm{GA}^{[11,12]}$.

\section{Methods}

A case of Gallbladder Adenomyomatosis treated with homoeopathic constitutional remedy. Ultrasonograghy results showed diffuse wall thickening $(8.2 \mathrm{~mm})$ of Gallbladder with obliteration of the lumen in some areas showing the characteristic hourglass appearance of Adenomyomatosis, which resolved after homoeopathic treatment and evaluated through abdominal USG.

\subsection{Case Summary}

A 17 year old girl presented with recurrent attack of upper abdominal pain of 1 year duration. Pain was aggravated by eating $(++)$, fried food, potato, fatty food and during night $(++)$. Pain relieved by lying on abdomen $(+++)$ and pressure.

\subsubsection{History of presenting complaint.}

Complaint started 2 years back as pain in abdomen which gradually increased in intensity. Took allopathic medicines from the medical college hospital Kottayam. Ultrasonography dated 24 /5 / 2018 showed gallbladder wall grossly thickened [8.1mm] showing an hourglass appearance and diagnosed as adenomyomatosis of gall bladder. No relief from allopathic medications for 1 year. History of recurrent attack of abdominal pain and intensity of pain also increased. Ultrasound scanning on 9 /4 /2019 reveals diffused gall bladder wall thickening [8.2mm] and hourglass appearance. Features were consistent with adenomyomatosis. Patient reported to our hospital on 10 /4 /2019 with severe pain in upper abdomen immediately after eating, especially after fatty food, fried items, pulses and tubers, and pain was more during night hours.

\subsubsection{Past History}

History of breathing difficulty in childhood and relieved after allopathic medications.

\subsubsection{Family History}

Mother suffering from allergic contact dermatitis.

\subsubsection{Physical Generals}

Appetite was increased. Patient desires cold food, sweets, chocolates and fried food. Thirst was reduced, desires cold water. Sweat was more on face and neck. Patient desires fanning and open air and desires bathing in cold water cold climate. Thermally hot.

Menstrual history: Menarche at the age of 13. Menses regular, last for 7 days, pain in lower abdomen, and extremities during first 2 days.

\subsubsection{Mental Generals:}

Weeps easily, like pets

\subsection{Evaluation \\ - Weeps easily \\ - Like pets \\ - Desire cold food \\ - Desires cold water \\ - Desire sweets \\ - Desire chocolates \\ - Desire fried food \\ - Desire open air \\ - Thermally hot \\ - Pain in upper abdomen \\ - < after eating \\ - < fried food \\ - $\quad<$ during night \\ - $\quad>$ lying on abdomen \\ - $>$ pressure}

\subsection{Remedy selection}

Phosphorus and Pulsatilla nigricans were the first two remedies on repertorisation. Patient was hot. So the chilly remedy was excluded. Pulsatilla was selected as the similimum on the basis of totality of symptoms (weeps easily, love for animals, desire for open air, thermally hot, desire cold food, desire cold drinks, desire sweets, desire chocolates, pain abdomen night aggravation, pain after eating aggravation). Pulsatilla 200 single dose was prescribed on 10/04/2019 and followed by placebo in the form of globules of 40 sizes, QID for one month.

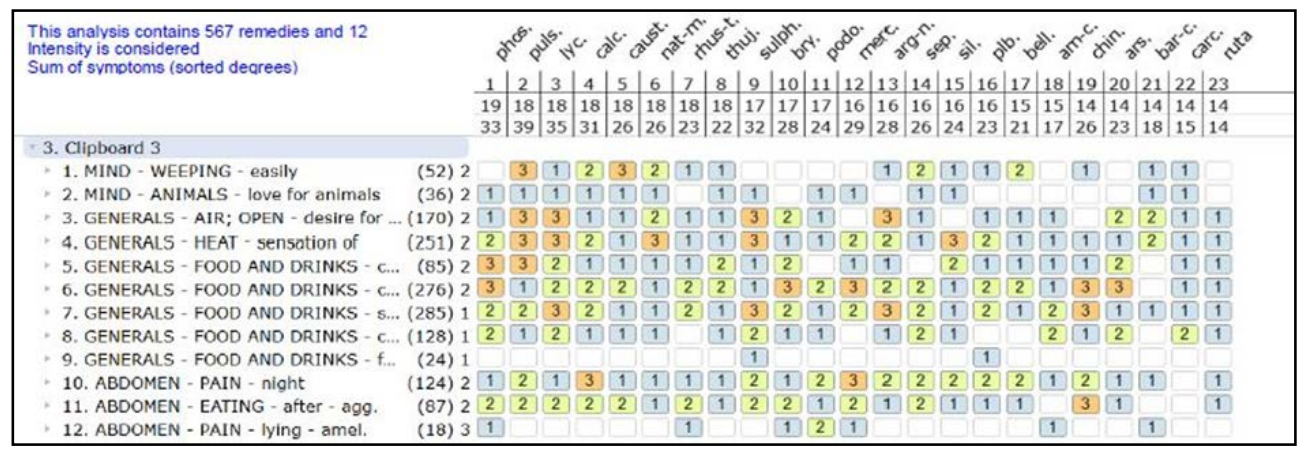

Fig 1: Repertorisation chart 
Table 1: Follow-up chart

\begin{tabular}{|c|c|c|c|}
\hline SI NO & Date & Observation & Prescription \\
\hline 1 & $11 / 5 / 2019$ & No history of pain for 3wks.Pain in upper abdomen++, after taking fatty food, night, $<$ 2days. & Pulsatilla 200/1 dose \\
\hline 2 & $8 / 6 / 2019$ & No history of pain till 7/6/19.but history of severe pain yesterday, sleeplessness due to pain. & Pulsatilla200/1dose \\
\hline 3 & $13 / 7 / 2019$ & No history of pain, sleep normal, appetite normal. & Placebo \\
\hline 4 & $10 / 8 / 2019$ & Pain after taking milk, intensity reduced, menses delayed. & Pulsatilla200/1dose \\
\hline 5 & $14 / 9 / 2019$ & Frequency and intensity of pain reduced, even after fattyfood. menses appeared. & Placebo \\
\hline 6 & $12 / 10 / 2019$ & Regular menses, no history of pain. & Placebo \\
\hline 7 & $9 / 11 / 201$ & Menses delayed for 2 weeks, dysmenorrhoea & Pulsatilla 1M/1dose \\
\hline 8 & $14 / 12 / 2019$ & No history of pain, thirst moderate, menses normal, not painful. & placebo \\
\hline 9 & $12 / 1 / 2020$ & No history of pain, thirst moderate, menses normal, not painful. & Placebo \\
\hline 10 & $9 / 2 / 2020$ & Pain in upper abdomen after taking meat and chicken puffs. & Pulsatilla 1M/1dose \\
\hline 11 & $8 / 3 / 2020$ & No history of pain, menses normal. & Placebo \\
\hline 12 & $10 / 5 / 2020$ & No history of pain, menses normal. & Placebo \\
\hline 13 & $12 / 7 / 2020$ & Breathing difficulty started for 1 week & Pulsatilla 10M/1dose \\
\hline 14 & $13 / 9 / 2020$ & No history of dyspnoea or dysmenorrhoea or abdominal pain. & Placebo \\
\hline 15 & $8 / 11 / 2020$ & Menses regular, No history of dyspnoea, or dysmenorrhoea or abdominal pain. & Placebo \\
\hline 16 & $10 / 1 / 2021$ & Menses regular, No history of dyspnoea, dysmenorrhoea or abdominal pain. & Placebo \\
\hline 17 & $14 / 3 / 2021$ & No history of abdominal pain, no dyspnoea, no dysmenorrhoea. & Placebo \\
\hline
\end{tabular}

\section{Results}

There was significant improvement after the first prescription itself and pain in upper abdomen was completely relieved after ten months of treatment. No history of abdominal pain after 9/2/2020 even after taking fatty food, fried items, potato or milk. Ultrasonography report on 16/4/2021 showed normal gallbladder distension with normal contour.

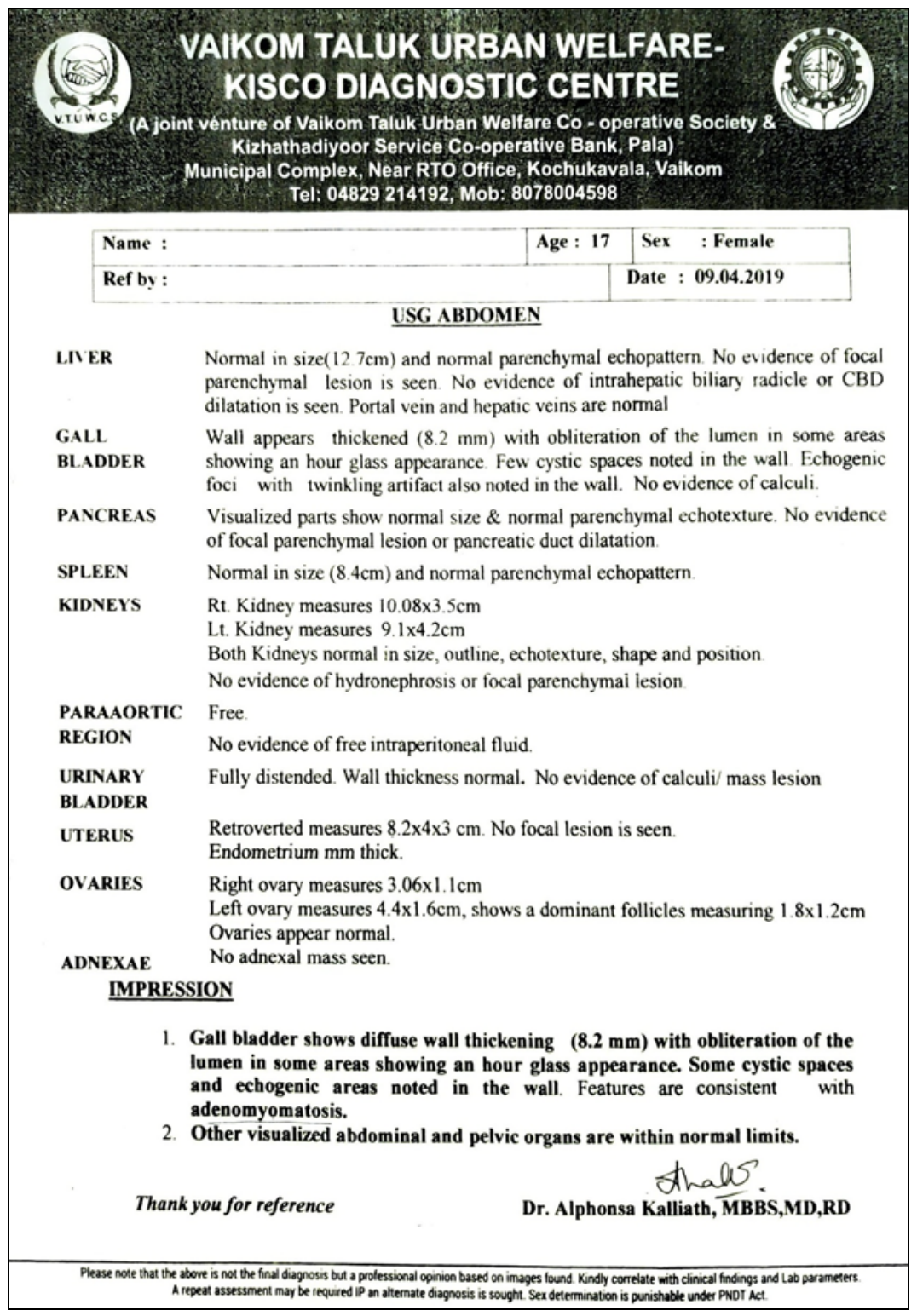

Fig 2: Ultrasound Report (2019) before starting Homoeopathic treatment 


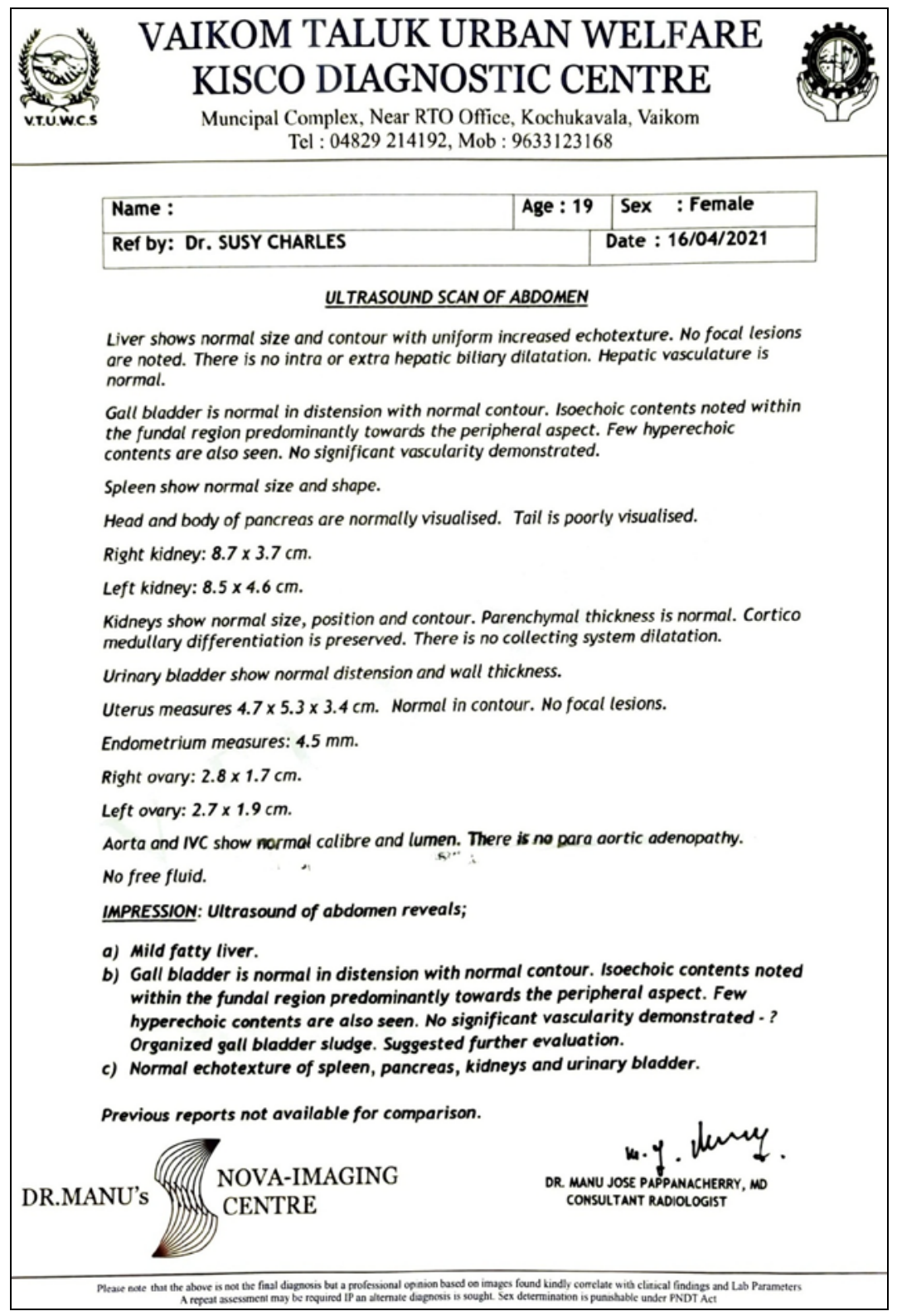

Fig 4: Ultrasound Report (2021) after Homoeopathic treatment

\section{Discussion}

Gallbladder adenomyomatosis (GA) is increasingly experienced in clinical practice due to increasing use of ultrasound imaging. Clinicians must know what are the consequences of this condition and its proper management [6]. The gallbladder wall is less than $2 \mathrm{~mm}$ thick in healthy subjects ${ }^{[13]}$. Mucosa, lamina propria, muscularis propria, and serosa are the four layers of gallbladder wall. Thickening of the wall in GA involves of both mucosa and muscularis propria ${ }^{[14]}$. GA is a degenerative as well as proliferative disease characterised by excessive epithelial proliferation and hypertrophy of muscularis propria.GA was first reported as precancerous condition by Albridge et al. and Nishimura et al. ${ }^{[18]}$. DNA in the mucosa of GA is similar to that of normal mucosa and GA contains no oncogene ${ }^{[4]}$.

Here, the case was diffuse type GA, rare form than fundal and segmental. Gallbladder showed diffuse wall thickening $(8.2 \mathrm{~mm})$ with obliteration of the lumen showing "hourglass appearance". After constitutional treatment with Pulsatilla 200 first followed by high potencies, gallbladder became in normal distension with normal contour. The constitutional medicine Pulsatilla not only relieved the abdominal pain but also bronchial asthma (childhood complaint) and dysmenorrhoea.

According to Dr. Hahnemann the homoeopathic physician should always be guided by the totality of symptoms in each case. He should never fall into the habit of making favourite medicines for nosologically labelled disease condition. A remedy is homoeopathic not by its peculiar method of preparation but by the mode of its application according to the principle of symptom similarity between the drug disease and the natural disease ${ }^{[20,21]}$.

\section{Conclusion}

Here a case of gallbladder adenomyomatosis was treated with the homeopathic remedy Pulsatilla. Pulsatilla was selected as the constitutional medicine on the basis of totality of symptoms after repertorisation. Recurrent attack of abdominal pain was relieved completely and ultrasound scan after treatment showed normal gallbladder contour. The successful management of the case proves the effectiveness of homoeopathic constitutional treatment in gallbladder adenomyomatosis. 


\section{References}

1. Golse N, et al. Gallbladder adenomyomatosis: Diagnosis and management. J Visc Surg 2017;154(5):345-353.

https://doi.org/10.1016/j.jvscurg.2017.06.004.

2. Norman Williams S, Christopher JK, Bulstrode P, Ronan O' Connell. Bailey and Love's Short Practice to Surgery. $25^{\text {th }}$ edition, Hodder Arrnold 2008, 1128.

3. Vikram Dogra S. Adenomyomatosis Imaging. emedicine.medscape.com 2018.

4. Pang L, Zhang Y, Wang Y, Kong J. Pathogenesis of Gallbladder adenomyomatosis: and its relationship with early-stage gallbladder carcinoma an overview. Braz J Med Biol Res 2018;51;(6):e7411.

5. Ram MD, Midha D. Adenomyomatosis of the Gallbladder. Surgery 1975;78(2):224-229.

6. Kit- Fai Lee, Esther HY Hung, Howard HW Leung, Paul BS Lai. A narrative review of gallbladder adenomyomatosis is what we need to know. Home/ Archives/ Annals of Translational Medicine 2020;8:23. https: //doi.org/ 10.21037/ATM-20-4897.

7. Cariati A, Celta F. Rokitansky- Aschoff sinuses of the gallbladder are associated with black pigment gallstone a scanning electron microscopy study. Ultrastructural Pathology 2002;27;(4):265-270. http://doi.org/10.1080/01913120309913.

8. Teelucksingh S, Welch T, Chan A, et al. Gallbladder Adenomyomatosis presenting with Abdominal Pain. Cureus 2020;12;(9):e10485. Doi:10.7759/cureus.10485.

9. Bonatti $\mathrm{M}$, et al. Gallbladder adenomyomatosis imaging findings, tricks and pitfalls, Insight Imaging April 2017;8(2):243-253. http://doi.org/10.1007/s13244-017-0544-7.

10. Kim BS, Oh JY, Nam KJ, et al. Focal thickening at the fundus of the gallbladder: computed tomography differentiation of fundal type adenomymatosis and localized chronic cholecystitis. Gut liver 2014;(8):219223. http;//doi.org/10.5009/gnl.2014.8.2.219.

11. Haaradome $\mathrm{H}$, et al. The pearl necklace sign: an imaging sign of adenomyomatosis of the gallbladder at MR cholangiopancreatography. Radiology 2003;227:80-88. http://doi.org/10.1148/radio.2271011378.

12. Lin SH, Chang FY, Yang YS, Jin JS, Chen TW. Rare gallbladder adenomyomatosis presenting as a atypical cholecystitis: case report BMC Gasteronterolology. 2011;11:106

13. Mahajan A, Sripathi S. Gallbladder Adenomyomatosis Mimicking Carcinoma: A Diagnostic Dilemma. J Glob Oncol 2;(5):341-5.

14. Alexis Boscak R, Mahmoud Al-Hawary, Stephen Ramsburgh R. Adenomyomatosis of the Gallbladder. Radiographics. pubs.rsna.org 2006;26:3.

15. Ryu Y, Bell D. Adenomyomatosis of the Gallbladder Radiology Reference Article radiopedia.org 2021.

16. Kai K, Ide $\mathrm{T}$, Masuda $M$, Kitahara K, Miyoshi A, Miyazaki $\mathrm{K}$, et al. Clinicopatological features of advanced gallbladder cancer assosciated with adenomyomatosis. Virchows Arch 2011;459(6):573-80.

17. Hammad AY, Miura JT, Turaga KK, Johnston FM, Hohenwalter MD, Gamblin TC. A literature review of radiological findings to guide the diagnosis of gallbladder adenomyomatosis. HPB (Oxford) 2016;18(2):129-35.
18. Nishimura A, Shirai Y, Hatayekama K. Segmental adenomyomatosis of the gallbladder predisposes to cholecytolithiasissis. J Hepatobiliary Pancreat Surg 2004;11(5):342-7.

19. Schroyens F. Synthesis Treasure Edition. Radar Opus 2009.

20. Hughes R. The Principle and Practice of Homeopathy. B. Jain Publishers Private Ltd New Delhi, India 1991.

21. Hahnemann S. Organon of Medicine $5^{\text {th }}$ and $6^{\text {th }}$ edition. B. Jain Publishers Private Ltd. New Delhi, India 1995. 